\title{
Methodological assessment of the reduction of dissemination risk and quantification of debris dispersion during dissection with a surgical aspirator
}

\author{
Sosuke Kageyama ${ }^{1}$, Atsuhiro Nakagawa ${ }^{1,2}$, Tomohiro Kawaguchi ${ }^{1,3^{*}}$ (1), Kiyonobu Ohtani ${ }^{4}$, Toshiki Endo ${ }^{1}$, \\ Manabu Kyan ${ }^{1}$, Tetsuya Kusunoki ${ }^{1}$, Yoshiteru Shimoda' ${ }^{1}$ Shin-Ichiro Osawa ${ }^{1}$, Masayuki Kanamori ${ }^{1}$, \\ Kuniyasu Niizuma ${ }^{1,5}$ and Teiji Tominaga ${ }^{1}$
}

\begin{abstract}
Objective: We developed an actuator-driven pulsed water jet (ADPJ) device to achieve maximal lesion dissection with minimal risk of normal structural damage. Despite the unique dissection characteristics, there is a risk of dissemination of tissue dispersion; however, there is no established method to quantify the dispersion. Hence, this study aimed to assess the factors associated with dispersion and propose a simple experimental method using spectrophotometry to evaluate the degree of dispersion in a wet field.

Results: Methylene blue-stained brain phantom gelatin was immersed in a chamber with distilled water solution and dissected with an ADPJ. The dispersed gelatin solution was stirred and warmed to dissolve. The absorbance of the solution was measured spectrophotometrically. First, a reference standard curve was constructed to confirm the relationship between the absorbance and the amount of the dispersed gelatin. A clear proportional correlation was observed, which indicated that absorbance measurements can help evaluate the amount of dispersion. Using this method, we revealed that a high dissection force, insufficient suction, and inappropriate long distance between the nozzle tip and the target were associated with increased dispersion. This method might constitute a versatile and reliable approach to evaluate dispersion and aid in the development of surgical devices.
\end{abstract}

Keywords: Dissemination risk, Debris dispersion, Pulsed water jet, Optical absorbance, Evaluation method

\section{Introduction}

Maximal resection of the target lesion without causing critical damage to the surrounding normal structures is one of the most important goals of surgical treatment in the field of neurosurgery. Removal of deep-seated lesions, such as intraventricular tumors, is the most challenging

\footnotetext{
*Correspondence: kawaguchi@nsg.med.tohoku.ac.jp

${ }^{1}$ Department of Neurosurgery, Tohoku University Graduate School

of Medicine, 1-1, Seiryo-machi, Aoba-ku, Sendai, Miyagi 980-8574, Japan
}

Full list of author information is available at the end of the article procedure owing to the deep location and proximity to the eloquent area [1, 2]. Neuroendoscopy is an emerging technology that holds promise because it causes minimal stress to the approach corridor. However, it is not easy to remove ventricle lesions with a flexible neuroendoscope alone because of restrictions in the instruments that can be applied simultaneously through the working channel [1-5]. New devices that can overcome these difficulties are the need of the hour to improve the effectiveness and safety of neuroendoscopic surgery $[1,2]$. 
We developed an actuator-driven pulsed water jet device (ADPJ) based on a new concept to achieve both maximal lesion dissection and minimal risk of normal structural damage [6]. Ejected water from the jet penetrates parenchymatous tissue, but vessels and neuronal fibers are preserved because of the differences in elasticity [7]. We reported the usefulness of ADPJ for ventricle dissection with vascular preservation based on tissue selectivity $[8,9]$. Although ADPJ has unique characteristics for tissue dissection, the issue of tissue dispersion occurs during dissection.

In this report, we assess the factors associated with dispersion during tissue dissection with ADPJ to reduce the risk of dissemination. At present, there is no established method to evaluate the maneuver-related dispersion of the dissected tissue. Therefore, we propose a simple evaluation method based on spectrophotometry.

\section{Main text \\ Method \\ Actuator-driven pulsed water jet}

The basic structure of ADPJ is shown in Fig. 1. Briefly, the piezoelectric actuator unit was attached to the end of the connecting pipe to drive the water (Fig. 1A). The pulsed water was ejected from the nozzle tip. The tip of the nozzle had an inner diameter of $0.15 \mathrm{~mm}$. The suction tube was set outside the connection pipe to remove excess water and splash. The detailed mechanism and device structure are described elsewhere [6].and then placed in the test chamber filled with $400 \mathrm{~mL}$ ofthe distilled water solution

\section{Brain phantoms}

Experimental brain phantoms were constructed with gelatin (BCN300S, Nitta Gelatin Co. Ltd., Osaka, Japan) at a concentration of $5 \% \mathrm{w} / \mathrm{v}$ [gelatin weight $(\mathrm{g}) /$ total solution volume $(\mathrm{mL})]$. To assess the dispersion of the debris, the brain phantom was stained with 0.82 weight percent (wt\%) methylene blue solution (Japan Pet Design Co. Ltd., Tokyo, Japan). The ratio of methylene blue to the total amount of gelatin was $0.123 \mathrm{wt} \%$ gelatin. The methylene blue solution was stirred and dissolved in warm distilled water at $65{ }^{\circ} \mathrm{C}$ and then cooled to $4{ }^{\circ} \mathrm{C}$ for $10 \mathrm{~h}$ for solidification.

\section{Brain phantom dissection in wet-field condition}

The brain phantom was removed from the storage buffer just before the experiments; $5 \mathrm{~mm}$ sections were trimmed off the surface to ensure an equal gelatin surface and then placed in the test chamber filled with $400 \mathrm{~mL}$ of the distilled water solution (Fig. 1B). The chamber containing the brain phantom was placed on a solid stage (EZSM 3E020K, Oriental Motor Co. Ltd., Tokyo, Japan) that moved horizontally at $0.5 \mathrm{~mm} / \mathrm{s}$. The ADPJ nozzle was set perpendicularly against the phantom surface. A pulsed water jet was ejected toward the brain phantom in the water chamber for $100 \mathrm{~s}$ (Fig. 1B). A high-speed camera (NX4S2, IDT Japan Co. Ltd., Tokyo, Japan) was set in front of the nozzle and operated at a rate of 5000 frames/s to observe the dispersion. The dissected brain phantom and the solution were used for further analyses.
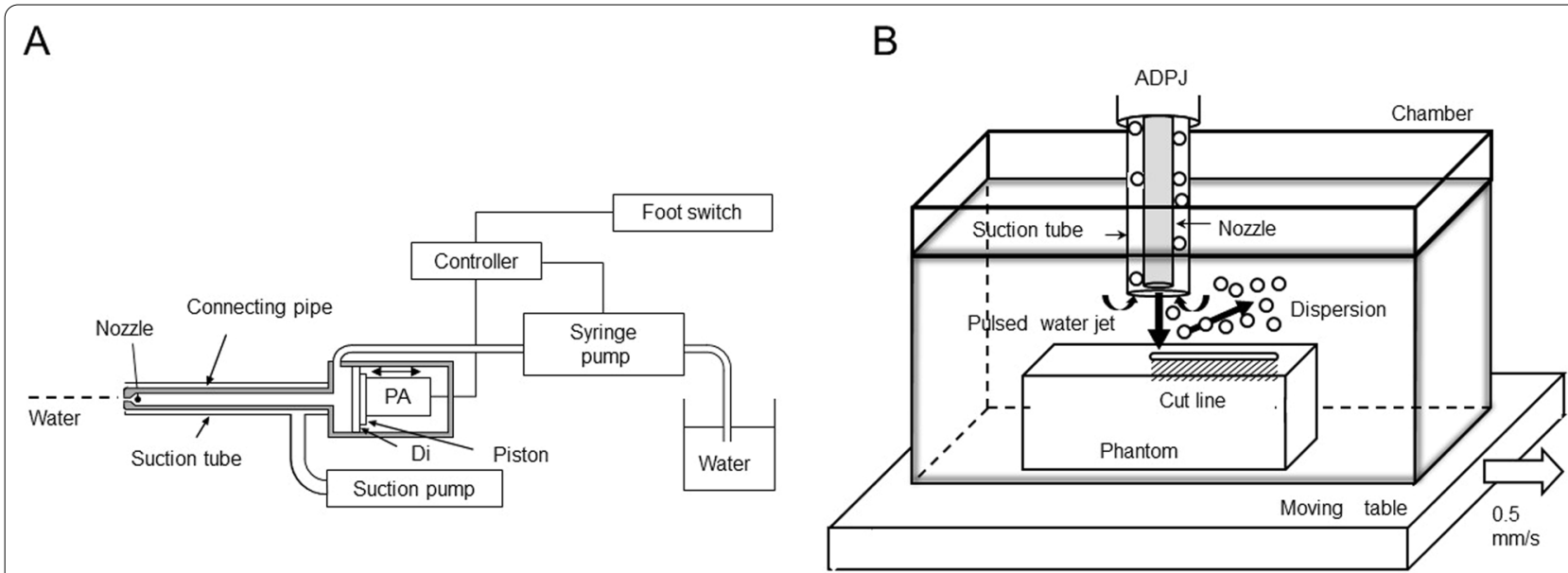

Fig. 1 Scheme of the piezo actuator-driven pulsed water jet (ADPJ) system (A). The water jet was generated by the movement of the driven piston and ejected from the nozzle tip. Di stainless steel diaphragm, PA piezo/electric actuator. B Scheme of brain phantom dissection in a wet-field condition. Dissected tissue and dispersion were aspirated through the suction tube placed outside the nozzle. Dispersion was recorded by high-speed camera 


\section{Factors associated with dispersion}

The relationship between the amount of dispersion and the following factors was analyzed: input voltage of the actuator, suction rate, and the distance between the nozzle and the target (standoff distance). The input voltage of the actuator ranged from 20 to $80 \mathrm{~V}$. The suction rate ranged from 0 to $200 \mathrm{~mL} / \mathrm{min}$. The standoff distance ranged from 1 to $2.5 \mathrm{~mm}$. The basic operating conditions are input voltage $60 \mathrm{~V}$, suction rate $200 \mathrm{~mL} / \mathrm{min}$, flow rate $7.6 \mathrm{~mL} / \mathrm{min}$, standoff distance $1.5 \mathrm{~mm}$, and frequency $400 \mathrm{~Hz}[6,10]$. No other condition was changed during the investigation of a parameter. All experiments were repeated 10 times for each parameter.

\section{Methylene blue absorbance}

Methylene blue is a near-infrared dye, with a maximum absorbance between 600 and $1000 \mathrm{~nm}$. All absorbance values were measured using an optical spectrophotometer (ASV11D, AS ONE Co. Ltd., Osaka, Japan) [11-13]. The methylene blue-stained solution was poured into a clear cuvette, and absorbance at $664 \mathrm{~nm}$ was measured. The measurements were repeated three times for each sample, and the mean was determined.

\section{Correlation between methylene blue absorbance and gelatin concentration}

To establish the relationship between the absorbance and the amount of dispersed gelatin, a reference standard curve was constructed. Seven pieces of the blue-stained gelatin, ranging from 20 to $400 \mathrm{mg}$, were precisely weighed using a laboratory balance $(\mathrm{Fx}-300 \mathrm{i}$, A\&D Co. Ltd., Tokyo, Japan) and dissolved in warmed water $\left(100 \mathrm{~mL}, 65^{\circ} \mathrm{C}\right)$. The absorbance at $664 \mathrm{~nm}$ was measured six times for each solution using a spectrophotometer. A clear proportional correlation was noted between absorbance and concentration, which indicated that measuring the absorbance of the solution can help in evaluating dispersion (Fig. 2).

\section{Measurement of dispersion after ADPJ dissection}

After dissection by ADPJ, the brain phantom was removed. Subsequently, the solution containing the dispersed gelatin was stirred and warmed to $65{ }^{\circ} \mathrm{C}$ for $10 \mathrm{~min}$ to dissolve the dispersed gelatin and equalize the concentration. The absorbance of the solution was measured spectrophotometrically, and the total dispersion was calculated using the standard curve described above.

\section{Statistical analysis}

Pearson product-moment correlation coefficient was calculated using JMP Pro 16 software (SAS Institute

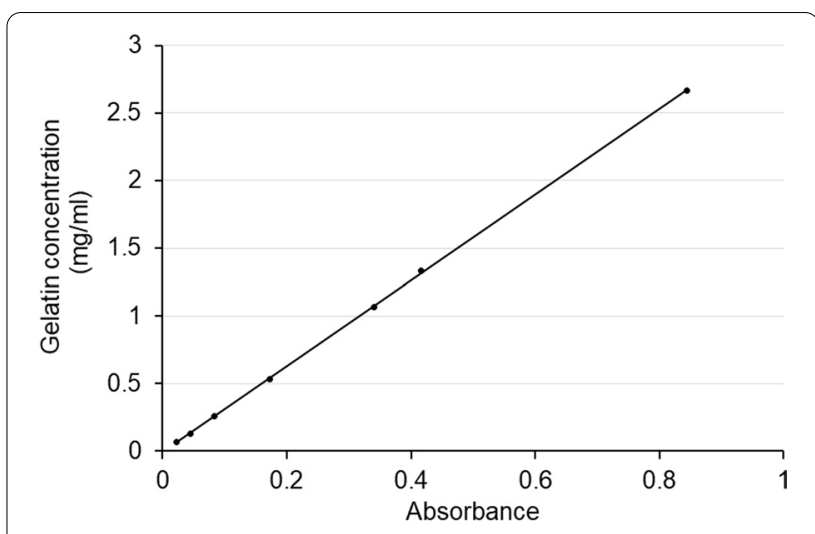

Fig. 2 Reference standard curve showing clear proportional correlation between absorbance and concentration

Inc., Cary, NC, USA). Statistical significance was set at a $\mathrm{p}<0.05$. In the correlation test, $\mathrm{r}$ showed the correlation between the $\mathrm{x}$ - and $\mathrm{y}$-axis. The absolute value of $r(|r|)$ denoted the strength of the correlation, and $|r|>0.7$ was defined as a strong correlation. All values were expressed as mean \pm standard deviation.

\section{Results}

\section{Dispersion and input voltage}

To evaluate the relationship between dispersion and ADPJ input voltage, we examined different voltages, i.e., $20,40,60$, and $80 \mathrm{~V}$. Other parameters remained the same as the basic operating conditions. With the setting of $20 \mathrm{~V}$, a small amount of dispersion was detected. The amount of dispersion increased as the input voltage was increased $(r=0.9534, p<0.05)$ (Fig. 3A).

\section{Dispersion amount and suction rate}

To assess the relationship between dispersion and ADPJ suction rate, we analyzed different suction rates, i.e., 0 , 50,100 , and $200 \mathrm{~mL} / \mathrm{min}$. Other parameters remained the same as the basic operating conditions. Without suction, the dispersion was maximum and, with a suction rate of $50 \mathrm{~mL} / \mathrm{min}$, the dispersion could not be controlled well. The amount of dispersion decreased with the increase in suction rate $(\mathrm{r}=-0.8683, \mathrm{p}<0.05)$ (Fig. 3B).

\section{Dispersion and standoff distance}

To investigate the relationship between dispersion and ADPJ standoff distance, we checked the different standoff distances, i.e., 1, 1.5, and $2.5 \mathrm{~mm}$. Other parameters remained as per the basic operating conditions. When the standoff distance was closer than $1 \mathrm{~mm}$, gelatin surface was distorted and damaged due to suction force. suction injury of the gelatin block itself occurred. The 

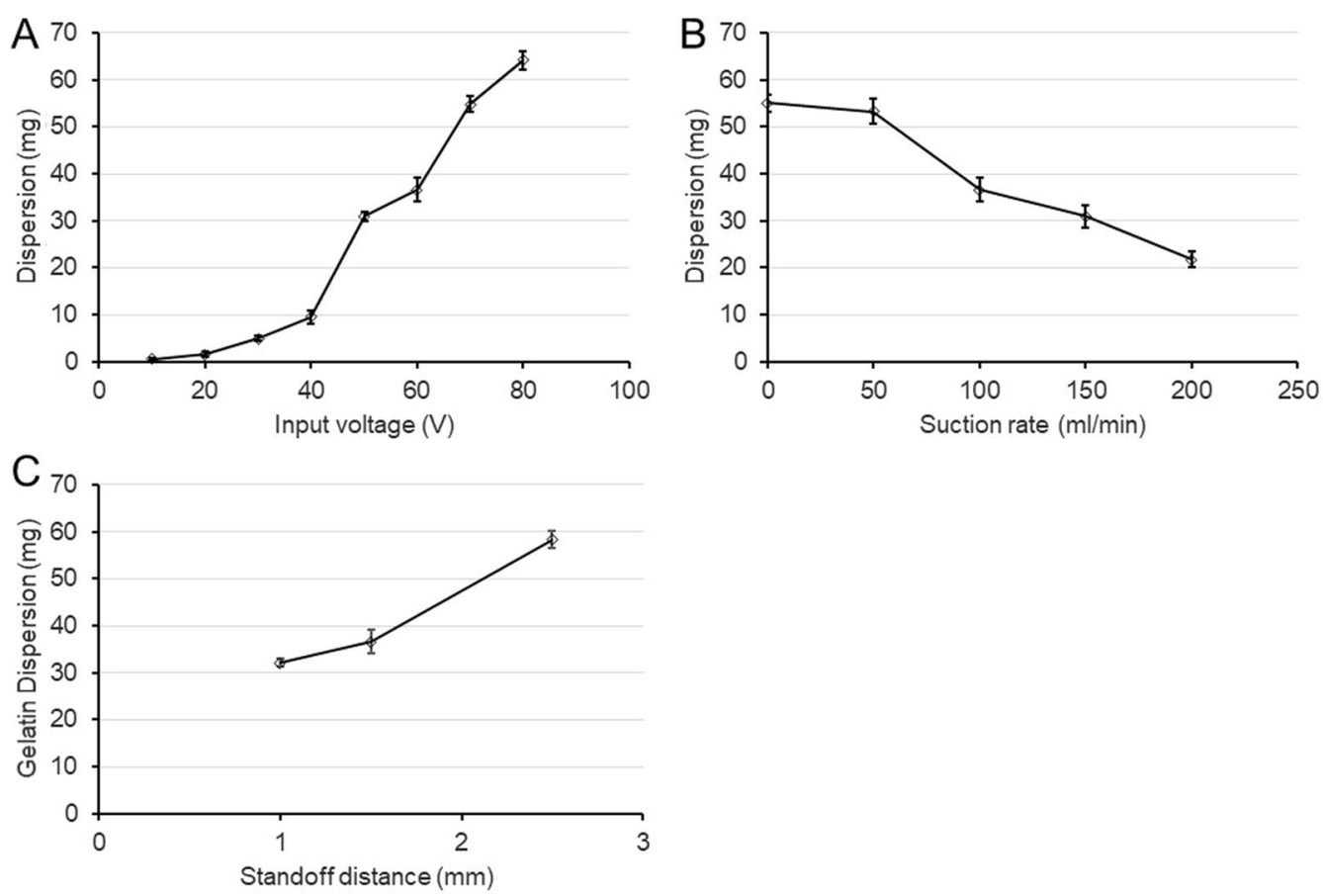

Fig. 3 A Graph showing the relationship between the input voltage and the amount of dispersion. B Graph showing the relationship between the suction rate and the amount of dispersion. C Graph showing the relationship between the standoff distance between the nozzle tip and the target and the amount of dispersion

greater the distance from the target, the higher was the dispersion $(r=0.8853, \mathrm{p}<0.05)($ Fig. 3C).

\section{Discussion}

Tremendous efforts have been taken to develop innovative devices that can improve surgical outcomes. New devices are designed based on unique concepts. Device safety and efficacy should be confirmed in an in vitro setting before applying it in an in vivo setting. Since improvement of the dissection profile is one of the most focused issues in the development of new dissection devices, the risk of minute tissue dispersion has not been well studied. Furthermore, there is no established method to evaluate dispersion. This study proposed and confirmed a simple quantitative method to assess tissue dispersion during surgical dissection in a wet field condition. According to this indicator, we assessed the factors associated with tissue dispersion.

We have been engaged in the development of new devices for more than 20 years. ADPJ is one of the most promising dissection devices and has achieved maximal resection with minimal risk of normal structural damage. In the process of in vivo experiments, we detected dispersing debris. Nakayashiki et al. estimated dispersion during dissection maneuvers by measuring the volume of splashed dots in two dimensions [14]. Although this evaluation method is unique and reliable, its application is limited to dry-field dissections. Moreover, small pieces of tissue might be dispersed in three dimensions during surgery.

To overcome such limitations in the evaluation of dispersion, we employed the technique used in basic research. In these fields, accurate measurement of the concentration of certain substances is quite important, but direct measurement is not always available. Thus, indirect methods are widely used. In our study, we dissected a gelatin-based brain phantom using ADPJ; hence, it was difficult to directly quantify the amount of dispersed gelatin in an in vitro setting. Thus, the brain phantom matrix was stained with methylene blue and, by measuring its absorbance, the concentration of the matrix was indirectly inferred.

This study revealed that both dissection force and suction rate are important factors in dispersion. Unsurprisingly, the brain phantoms were dissected more deeply with the use of higher input voltage and greater dispersion. Moreover, a higher suction rate was associated with less dispersion. To achieve effective dissection, adequate dissection force is necessary, and mickle dispersion is inevitable; therefore, strong aspiration is required. Our results suggest that an appropriate standoff distance between the nozzle tip and the target is crucial since a 
short distance may injure the tissue, while a long distance might increase the risk of dispersion.

\section{Conclusions}

We have described a simple method for evaluating tissue dispersion during surgical maneuvers, which has not been previously established. For the development of new dissection devices, dispersion control is one of the key issues to alleviate the risk of dissemination. Appropriate suction systems can effectively avoid surgical maneuverrelated dissemination even with valid dissection devices, such as cavitron ultrasonic surgical aspirator (CUSA) and ADPJ.

\section{Limitations}

The present study has several limitations. First, the surface of the gelatin brain phantom can be dissolved in the solution without dissection during the experiment. A control phantom without ADPJ dissection was immersed in the solution for the same duration as the dissection experiment, and spectrophotometry revealed that elution was very low. Hence, no special consideration was required (data not shown). Second, the suction rate was higher than the ADPJ flow rate, so the volume of the solution in the chamber decreased during the experiment. Thus, we chose $100 \mathrm{~s}$ as the duration of dissection to avoid drying up of the solution. In a clinical setting, alteration of intracranial pressure by the excessive drainage of cerebrospinal fluid should be taken into consideration. Third, there might be measurement errors associated with spectrophotometry. Our method did not amplify the signals; hence, the absorbance value was not high with minute deviations, which resulted in reliable results. In fact, the standard curve showed a good correlation between the gelatin concentration and the methylene blue absorbance (Fig. 2). Fourth, although we showed that higher input voltage was associated with increased dispersion, the dissection efficacy was not assessed. In a clinical setting, the maximal dissection of the target lesion is the primary purpose of the surgery. To achieve both effective dissection and a low dispersion rate, further optimization of the ADPJ condition is required. Finally, our method to evaluate dispersion was confirmed by ADPJ only, and there is no comparable data with other dissection devices such as CUSA. The setting condition is completely different among devices, and the results cannot be compared directly. Collecting more data and confirming the findings are planned in the future to strengthen the reliability.

\section{Abbreviations}

ADPJ: Actuator-driven pulsed water jet; CUSA: Cavitron ultrasonic surgical aspirator.

\section{Acknowledgements}

The authors acknowledge Emiko Kaneda for technical support in data collection. The authors also acknowledge Emiko Kaneda, Kyoko Sugai, Naoko

Terashima, and Mari Komuro for administrative assistance.

\section{Authors' contributions}

Concept/design: SK, ToK, and AN. Data analysis/interpretation: AN, TT. Data Acquisition: SK, KO, TE, MK, TeK, YS, SO, MK, and AN. Drafting of the article: SK, TK1, and AN. Critical revision of the article:TK1, AN, KN, and TT. All authors read and approved the final manuscript.

\section{Funding}

This study was supported by the following funds: Grant-in-Aid for Scientific Research (A) 18H04157; Grant-in-Aid for Scientific Research (B) 19H03755; Grant-in-Aid for Scientific Research (C) 20K12057, 19K08090, 18K08561,18K08932, 18K08960; Grant-in-Aid for challenging Exploratory Research, 16K15810, from the Japanese Ministry of Education, Culture, Sports, Science, and Technology (MEXT). This research was supported by AMED under Grant Number_JP19hm0102064_. Dr. Nakagawa and Dr. Tominaga received research support for this study from Seiko Epson Co., Ltd., under a collaborative research contract with Tohoku University. This study was funded in part by a grant from the Japanese Foundation for Research and Promotion of Endoscopy. The Translational Research Network Program and Dr. Kawaguchi, Dr. Nakagawa, and Dr. Tominaga are patent holders for technologies with Seiko Epson Co., Ltd., including the supply of the piezo ADPJ system. The funding body had no role in study design, collection, analysis, interpretation of the data, or drafting of the manuscript.

\section{Availability of data and materials}

The datasets used and/or analyzed during the current study are available from the corresponding author upon reasonable request.

\section{Declarations}

Ethics approval and consent to participate Not applicable.

Consent for publication

Not applicable.

\section{Competing interests}

The ADPJ device was a free rental from Seiko Epson Corporation under a collaborative research contract with Tohoku University to T.K, T.K., and A.N.

\section{Author details}

${ }^{1}$ Department of Neurosurgery, Tohoku University Graduate School of Medicine, 1-1, Seiryo-machi, Aoba-ku, Sendai, Miyagi 980-8574, Japan. ${ }^{2}$ Department of Biodesign, Clinical Research, Innovation, Education Center, Tohoku University Hospital, Sendai, Miyagi, Japan. ${ }^{3}$ Department of Neurosurgery, Kohnan Hospital, Sendai, Miyagi, Japan. ${ }^{4}$ Institute of Fluid Science, Tohoku University, Sendai, Miyagi, Japan. ${ }^{5}$ Department of Neurosurgical Engineering and Translational Neuroscience, Tohoku University Graduate School of Medicine, Sendai, Miyagi, Japan.

Received: 6 October 2021 Accepted: 2 February 2022

Published online: 02 March 2022

References

1. Marcus HJ, Seneci CA, Payne CJ, Nandi D, Darzi A, Yang GZ. Robotics in keyhole transcranial endoscope-assisted microsurgery: a critical review of existing systems and proposed specifications for new robotic platforms. Neurosurgery. 2014;10(Supplement 1):84-95.

2. Snyderman CH, Carrau RL, Prevedello DM, Gardner P, Kassam AB. Technologic innovations in neuroendoscopic surgery. Otolaryngol Clin North Am. 2009;42(5):883-90.

3. Albright AL, Okechi H. Use of the NICO Myriad device for tumor and cyst removals in a developing country. Childs Nerv Syst. 2012;28(4):599-604. 
4. Dlouhy BJ, Dahdaleh NS, Greenlee JD. Emerging technology in intracranialneuroendoscopy: application of the NICO Myriad. Neurosurg Focus. 2011;30(4):E6.

5. McLaughlin N, Ditzel Filho LF, Prevedello DM, Kelly DF, Carrau RL, Kassam AB. Side-cutting aspiration device for endoscopic and microscopic tumor removal. J Neurol Surg B Skull Base. 2012;73(1):11-20.

6. Seto T, Yamamoto H, Takayama K, Nakagawa A, Tominaga T. Characteristics of an actuator-driven pulsed water jet generator to dissecting soft tissue. Rev Sci Instrum. 2011:82(5):055105.

7. Nakagawa A, Kumabe T, Kanamori M, Saito R, Hirano T, Takayama K, et al. Clinical application of pulsed laser-induced liquid jet: preliminary report in glioma surgery. No Shinkei Geka. 2008;36(11):1005-10.

8. Kawaguchi T, Nakagawa A, Endo T, Fujimura M, Sonoda Y, Tominaga T. Ventricle wall dissection and vascular preservation with the pulsed water jet device: novel tissue dissector for flexible neuroendoscopic surgery. $J$ Neurosurg. 2016;124(3):817-22.

9. Kusunoki T, Kawaguchi T, Nakagawa A, Noguchi Y, Osawa SI, Endo H, et al. Effect of endoscope flexibility on tissue dissection profile assessed with pulsed water jet device: ensuring safety, efficacy, and handling of thin devices for neuroendoscopic surgery. BMC Res Notes. 2021:14(1):64.

10. Sato Y, likubo M, Nishioka T, Yoda N, Kusunoki T, Nakagawa A, et al. The effectiveness of an actuator-driven pulsed water jet for the removal of artificial dental calculus: a preliminary study. BMC Oral Health. 2020;20(1):205

11. Bessho M, Furuta M, Kojima T, Okuda S, Hara M. Gelatin hydrogels crosslinked by gamma-ray irradiation: materials for absorption and release of dye. J Biomater Sci Polym Ed. 2005;16(6):715-24.

12. Osathanunkul M, Buddhachat K, Chomdej S. A modified colorimetric method of gelatinolytic assay using bacterial collagenase type II as a model. Anal Biochem. 2013;433(2):168-70.

13. Nethery A, Lyons JG, O'Grady RL. A spectrophotometric collagenase assay. Anal Biochem. 1986;159(2):390-5.

14. Nakayashiki A, Kawaguchi T, Nakagawa A, Sato M, Mochizuki F, Endo $T$, et al. Water veil effect to control splashing from the pulsed water jet device: minimizing the potential risk of dissemination using surgical aspirators. J Neurol Surg A Cent Eur Neurosurg. 2018;79(4):309-15.

\section{Publisher's Note}

Springer Nature remains neutral with regard to jurisdictional claims in published maps and institutional affiliations.

Ready to submit your research? Choose BMC and benefit from:

- fast, convenient online submission

- thorough peer review by experienced researchers in your field

- rapid publication on acceptance

- support for research data, including large and complex data types

- gold Open Access which fosters wider collaboration and increased citations

- maximum visibility for your research: over $100 \mathrm{M}$ website views per year

At BMC, research is always in progress.

Learn more biomedcentral.com/submissions 